\title{
Photoinduced Increase in Vesicle Size and Role of Photoresponsive Malachite Green Leuconitrile Derivative in Vesicle Fusion
}

\author{
Ryoko M. Uda, Daisuke Yamashita, Yoshiaki Sakurai, ${ }^{\dagger}$ and Keiichi Kimura ${ }^{*}{ }^{\ddagger}$ \\ Department of Chemical Engineering, Nara National College of Technology, Yata 22, Yamato- \\ koriyama, Nara 639-1080, Japan
}

Technology Research Institute of Osaka Prefecture, Ayumino 2-7-1, Izumi, Osaka 594-1157, Japan

Department of Applied Chemistry, Faculty of Systems Engineering, Wakayama University, Sakae-dani 930, Wakayama 640-8510, Japan

kkimura@sys.wakayama-u.ac.jp

\section{Photoionization ratio of the Malachite Green leuconitrile derivative}

The ionization ratio is defined by $A_{M G L} / A_{M G O}$, where $A_{M G L}$ and $A_{M G O}$ are the absorbance at $615 \mathrm{~nm}$ of $1.68 \times 10^{-5} \mathrm{~mol} \cdot \mathrm{dm}^{-3} 1$ in vesicle dispersion after UV irradiation and $1.68 \times 10^{-5} \mathrm{~mol} \cdot \mathrm{dm}^{-3}$ Malachite Green oxalate in acetate buffer solution respectively. 


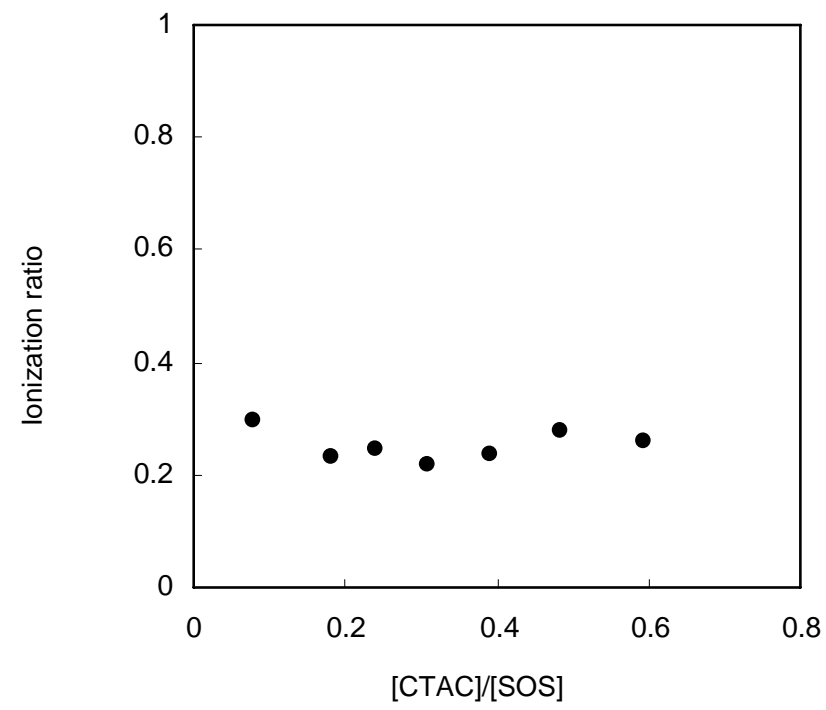

Figure S1. Dependence of photoionization ratio of Malachite Green leuconitrile derivative on the ratio of [CTAC]/[SOS].

\section{Quenching ANTS fluorescence by DPX in solutions}

Because DPX quenches ANTS fluorescence by collisional transfer, the relationship between ANTS and DPX concentrations was examined in aqueous solutions. The relation indicates the dilution effect of DPX on quenching ANTS fluorescence, therefore the quenching of ANTS fluorescence caused by the vesicle fusion in Figure 9 is distinguished from that caused by the simple dilution of DPX which is released from the vesicle to aqueous medium.

The fluorescence intensity of ANTS was plotted against that of the ANTS solution with the 16-fold concentration of DPX (Figure S2). Various fluorescence intensities were obtained by diluting the two stock solutions: (1) $1.0 \times 10^{-4} \mathrm{~mol} \cdot \mathrm{dm}^{-3}$ ANTS and (2) $1.0 \times 10^{-4} \mathrm{~mol} \cdot \mathrm{dm}^{-3}$ ANTS and $1.6 \times 10^{-3}$ mol $\cdot \mathrm{dm}^{-3}$ DPX. The fluorescence scale was calibrated with the fluorescence of the stock solution (1). The plots on the dotted line in Figure S2 indicate that the fluorescence of ANTS is not quenched by DPX at the fluorescence intensity. When the intensity is greater than $20 \%$, the fluorescence intensity of the ANTS solution containing DPX is smaller than that of the ANTS solution, and the quenching of the ANTS fluorescence cannot be negligible. However, the fluorescence intensity in Figure 9 is smaller than 13\%, and therefore the concentration of DPX is too small to quench the ANTS fluorescence. When the ANTS- and DPX-encapsulating vesicles were destabilized to release the ANTS and DPX into the medium and thereby cause their dilution, the quenching of ANTS did not take place. Therefore, the fusion process keeps the DPX concentration high enough to quench ANTS fluorescence, thus being 
responsible for the quenching of ANTS fluorescence by DPX at any fluorescence intensity smaller than 13\% (Figure 9).

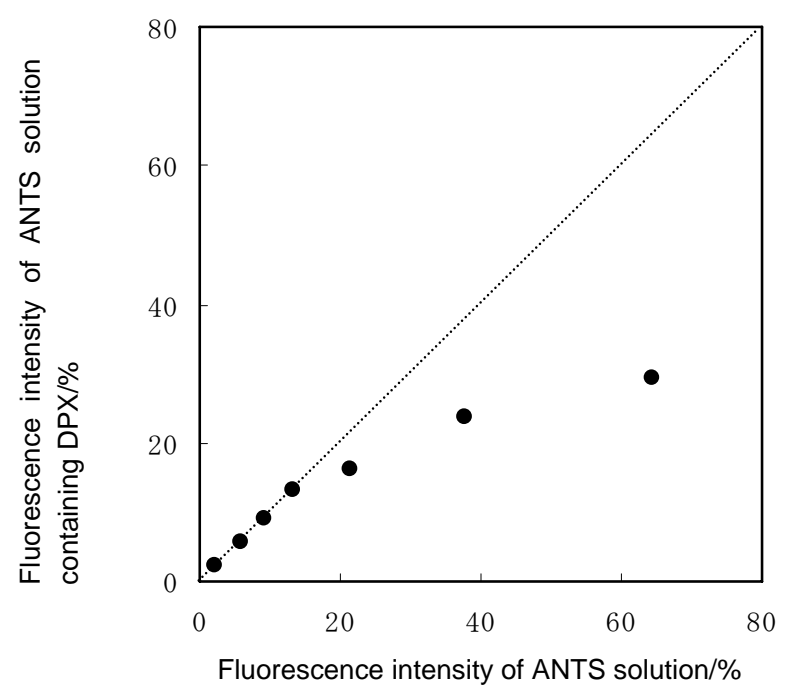

Figure S2. Relationship between fluorescence intensity of ANTS and that with the 16-fold concentration of DPX. $100 \%$ fluorescence was obtained with $1.0 \times 10^{-4} \mathrm{~mol} \cdot \mathrm{dm}^{-3}$ of ANTS at $507 \mathrm{~nm}$ in acetate buffer $(\mathrm{pH}=4.0)$.

\section{Fluorescence emission spectrum of pyrene in tetrahydrofuran}

The tetrahydrofuran (THF) solution containing $10^{-4} \mathrm{~mol} \cdot \mathrm{dm}^{-3}$ pyrene and $10^{-2} \mathrm{~mol} \cdot \mathrm{dm}^{-3} \mathbf{1}$ was excited at $337 \mathrm{~nm}$, and the resulting emission spectra were recorded. The peak around 485nm was observed and exciplex formation was confirmed in the organic solvent (Figure S3). 


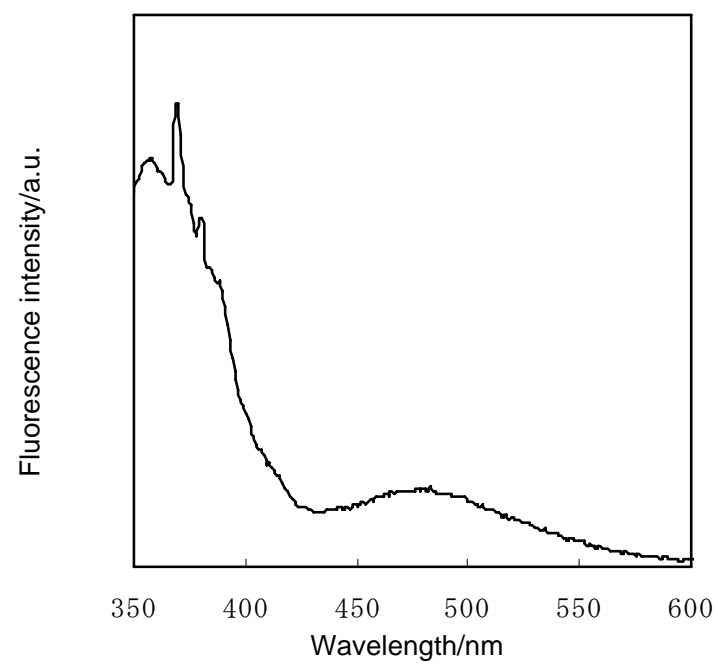

Figure S3. Fluorescence emission spectra of pyrene $\left(10^{-4} \mathrm{~mol} \cdot \mathrm{dm}^{-3}\right)$ in tetrahydrofuran solution containing $1\left(10^{-2} \mathrm{~mol} \cdot \mathrm{dm}^{-3}\right)$. Excited wavelength is $337 \mathrm{~nm}$. 